\title{
Des exploitations intensives d'huîtres pendant l'Antiquité et le Moyen Âge sur le littoral atlantique français: l'exemple de Beauvoir-sur-Mer (Vendée)
}

\author{
Catherine DUPONT \\ UMR 6566 CReAAH Centre de Recherche en Archéologie, Archéosciences, Histoire, \\ CNRS, Universités de Rennes 1, Rennes 2, Nantes, Le Mans, Ministère de la Culture, INRAP, \\ Université de Rennes 1, Campus de Beaulieu, Bâtiment 24-25, CS 74205, \\ F-35042 Rennes cedex (France) \\ catherine.dupont@univ-rennes1.fr \\ Nicolas ROUZEAU \\ Service régional de l'Archéologie, DRAC Provence Alpes Côte d'Azur, \\ 23 boulevard du Roi René, F-13617 Aix-en-Provence cedex 1 (France) \\ nicolas.rouzeau@culture.gouv.fr
}

Publié le 31 décembre 2015.

MOTS CLÉS

Ostrea edulis,

huître plate,

Moyen Âge,

spécialisation,

paléoenvironnement.
Dupont C. \& Rouzeau N. 2015. - Des exploitations intensives d'huîtres pendant l'Antiquité et le Moyen Âge sur le littoral atlantique français: l'exemple de Beauvoir-sur-Mer (Vendée). Anthropozoologica 50 (2): 109-122. http://dx.doi. org/10.5252/az2015n2a4

\section{RÉSUMÉ}

La consommation des huîtres plates Ostrea edulis Linnaeus, 1758 est connue dès le Mésolithique le long de la façade atlantique européenne (Gutiérrez-Zugasti et al. 2011). Ce constat se vérifie également sur les côtes françaises (Dupont 2006). L'exploitation de ce coquillage est très importante pendant l'Antiquité (Ferdière 1988). Avec le développement du transport de marchandises, ce mollusque est alors découvert sur des sites archéologiques éloignés de plusieurs centaines de kilomètres du trait de côte. Le succès de l'huître se poursuit pendant le Moyen Âge. D'importantes accumulations de ce bivalve sont fréquemment découvertes dans des amas coquilliers le long du littoral atlantique français. Certains d'entre eux sont mieux connus car ils se matérialisent sous la forme de hautes buttes qui se démarquent dans le paysage. Beaucoup d'autres sont ignorés car ils se situent actuellement sous d'anciens villages côtiers. Ces sites archéologiques où dominent les coquillages font rarement l'objet de fouilles. L'étude proposée, qui traite du site de Beauvoir-sur-Mer (Vendée), est, à ce titre, une nouveauté en la matière. Ce site se caractérise par un énorme amas coquillier d'une surface de $1700 \times 25 \mathrm{~m}$ et de 1-2 m d'épaisseur. Sa formation est datée entre le VIIe et le XIVe siècles. Dans sa proximité un château a été érigé au XIIIe siècle. Il apparaît au vu des données de terrain que l'accumulation de coquilles s'est faite avant l'édification du château et a perduré lors de son occupation. Pour la présente analyse, un échantillon de 5673 coquilles a été étudié. Elle montre comment ces huîtres ont été exploitées sur le littoral, dans quel type d'environnement, comment elles ont été sélectionnées... L'analyse biométrique des huîtres associée à l'observation des stigmates d'ouverture montre que les plus grandes huîtres ont été ouvertes et que la chair en a été extraite immédiatement. Si ce type d'exploitation des huîtres est connu pour le Moyen Âge, des fouilles récentes au Langon montrent que cette extraction intensive de la chair des huîtres a pu débuter dès l'Antiquité. De plus, la faune associée identifiée à Beauvoir-sur-Mer nous informe sur la zone du littoral où les huîtres ont été exploitées, ainsi que sur le type de biotope dans lequel elles ont été collectées. Les résultats obtenus pour ce site sont confrontés à d'autres accumulations historiques d'huîtres dans la région. 


KEY WORDS
Ostrea edulis,
flat oyster,
Middle Ages,
specialization,
palaeoenvironment.

\begin{abstract}
Intensive exploitation of oysters during Antiquity and the Middle Ages on the French Atlantic coast: the example of Beauvoir-sur-Mer (Vendée).

Along the European Atlantic facade, the consumption of flat oysters (Ostrea edulis Linnaeus, 1758) has been known since the Mesolithic era (Gutiérrez-Zugasti et al. 2011). This observation is also true for French coasts (Dupont 2006). Especially during Antiquity, the exploitation of this species became extensive (Ferdière 1988). With the development of transport facilities, oysters were found at archaeological sites located hundreds of kilometers away from the coast. This is a clear hint of their success that lasted into the Middle Ages. Along the Atlantic coast, large accumulations of oysters are recovered from so-called middens, some of which are well-known because they form conspicuous land-marks. However, the majority of such middens are thought to be hidden under modern villages. In France, archaeological sites where marine shells - in particular oysters - form the majority of consumption refuse, are only rarely excavated. Therefore, the present study about Beauvoir-sur-Mer (Vendée) is rather novel. The site can be characterized as an enormous shell midden $1700 \times 25 \mathrm{~m}$ in size and 1-2 $\mathrm{m}$ thick. It is dated to between the $7^{\text {th }}$ and $14^{\text {th }}$ centuries AD. Near to the site, a castle was constructed during the $13^{\text {th }}$ century $\mathrm{AD}$. The formation of the shell midden began before, and continued during the castle occupation. For the current analysis, a sample of 5673 shell remains was studied. The analysis presented here, mainly focusses on how the oysters were exploited on the coast, in what type of environment, how they were selected etc. Biometrical analyses linked to the observation of traces produced from the opening of the bivalves, showed in particular that bigger oysters were opened on-site and their flesh was extracted immediately. This way of handling oysters was known from the Middle Ages. However, actual archaeological research at Le Langon shows that this could well have been practiced ever since Antiquity. The associated fauna identified at Beauvoirsur-Mer informs us about the range where the oysters have been exploited and the kind of biotope in which they were gathered. The results gained for this site are compared to accumulations of oysters from other historic sites in the region.
\end{abstract}

\section{INTRODUCTION}

L'étude des coquillages en contexte archéologique est une discipline relativement nouvelle en France. Cette caractéristique rend difficile l'intégration des études malacofauniques dans des contextes chronologiques larges et limite les comparaisons. Pour le site de Beauvoir-sur-Mer (Vendée), qui sera présenté plus en détail dans ce qui suit, l'étude des coquillages n'a pu être réalisée que 21 ans après sa fouille. Ce fait est lié à la rareté de spécialistes dédiés à l'archéomalacologie lors de la fouille. Il faut également souligner le caractère exceptionnel de cette opération de terrain, dont le principal artefact découvert reste la coquille. Cette caractéristique rend malheureusement sa datation peu précise, bien que les données de terrain permettent de cibler sa formation entre les VIIe et XIVe siècles. Le volume que composent ces mollusques est important. Il s'étend sur plus de 1000 mètres pour une épaisseur qui avoisine parfois les 2 mètres. Bien qu'il soit invisible dans le paysage, son important volume et sa composition majoritaire en restes coquilliers permet de le nommer amas coquillier (Dupont 2006). Le terme de dépôt coquillier lui est préféré lorsque les volumes coquilliers découverts sont inférieurs à $2 \mathrm{~m}^{3}$ (Dupont 2006). Majoritairement composé de valves d'huîtres, dont beaucoup sont encore jointives, il nous paraissait intéressant de confirmer l'origine anthropique de l'accumulation de Beauvoir-sur-Mer. Leur analyse minutieuse permet également de recomposer certains des gestes techniques qui ont accompagné leur exploitation sur le littoral, mais aussi sur leur lieu de traitement. Chacune de ces étapes du traitement des huîtres a été étudiée de façon systématique, appuyée par une méthodologie novatrice et adaptée aux spécificités du site de Beauvoir-sur-Mer.

Bien que délaissées par l'archéologie, ces accumulations composées quasi-exclusivement d'huîtres sont loin d'être rares sur le littoral atlantique de l'ouest de la France. Les résultats obtenus à Beauvoir-sur-Mer sont comparés aux sites similaires afin de mieux saisir le type d'activité qui se cache derrière ses immenses accumulations de coquillages.

\section{BEAUVOIR-SUR-MER: UN SITE ÉNIGMATIQUE}

\section{Historique DES RECHERCHES SUR LE SITE}

DE BEAUVOIR-SUR-MER

C'est en 1986, date du décret relatif à la préservation du patrimoine archéologique, que le Service Régional de l'Archéologie des Pays de la Loire a délimité la totalité de l'accumulation d'huîtres de Beauvoir-sur-Mer par arrêté préfectoral. Ce service, en la personne de $\mathrm{N}$. Rouzeau, est ensuite intervenu à cinq reprises en vue d'évaluer la problématique d'étude de ce site archéologique. Par la suite, la découverte récurrente de dépôts coquilliers volumineux, comme par exemple l'identification d'un amas d'huîtres au Langon (Vendée) décrit par le Dr. Baudoin en 1912 lors de sondages effectués par F. Guérin (INRAP), a poussé $\mathrm{N}$. Rouzeau à prescrire dans les cahiers des charges d'opérations archéologiques, le recrutement spécifique d'un malacologue 


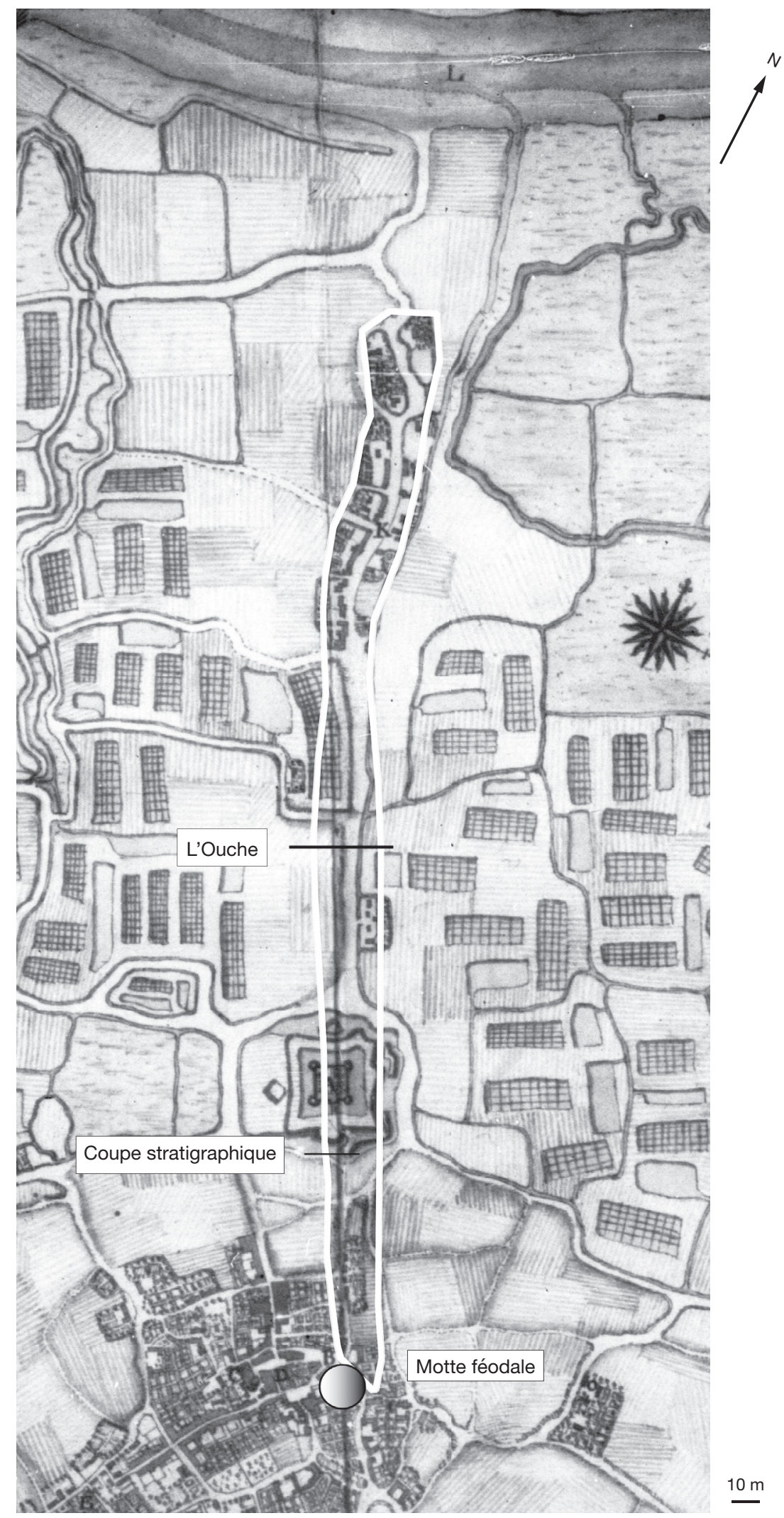

FIG. 1. - Extension de l'amas d'huîtres de Beauvoir-sur-Mer reportée sur le plan de 1685 (N. Rouzeau, SRA). 

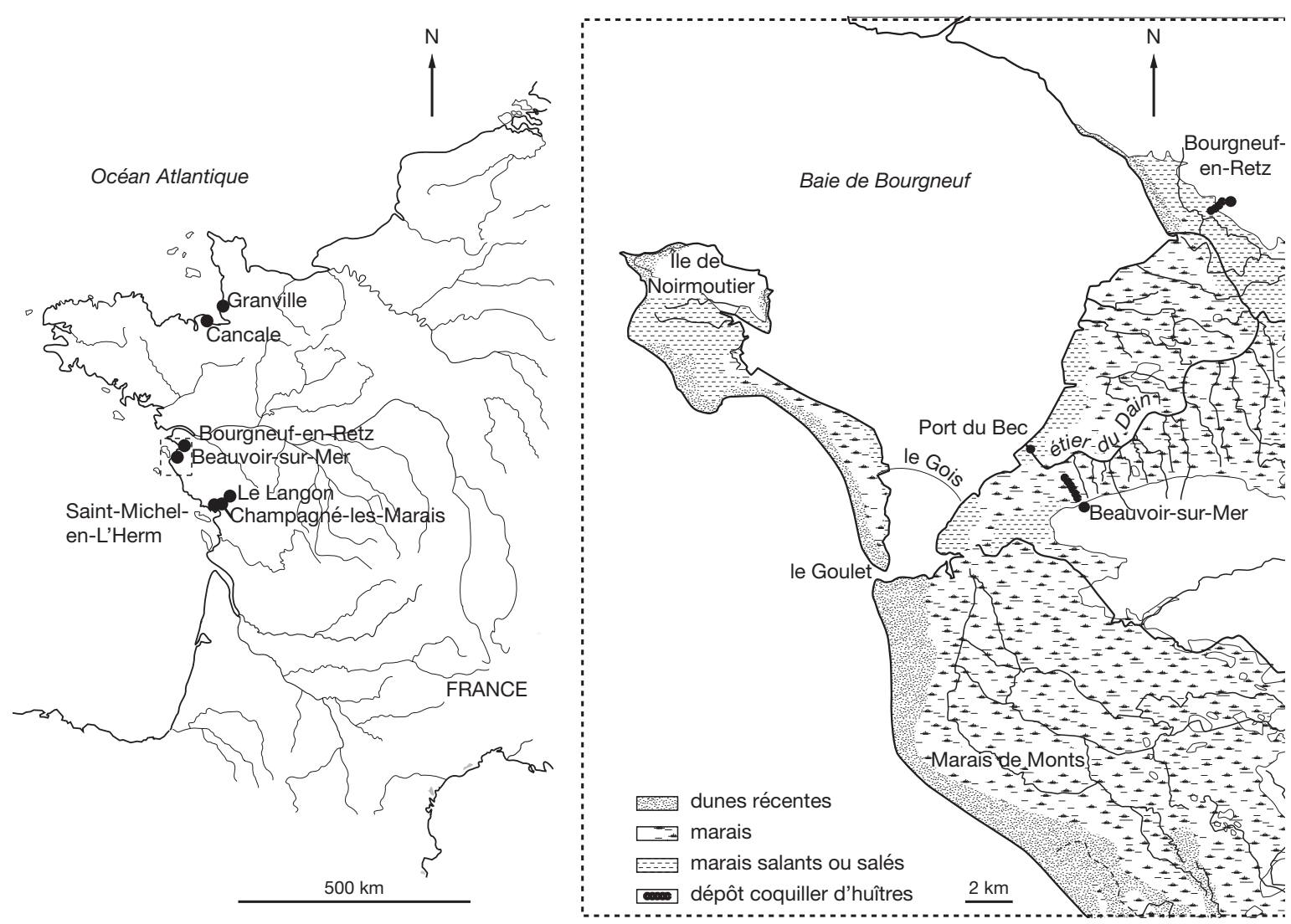

FIG. 2. - Localisation des dépôts coquilliers mentionnés dans le texte, d'après Verger 2005 (L. Quesnel, CNRS).

comme co-responsable des chantiers de fouille. Cette présence sur le terrain permet d'intégrer les problématiques liées aux coquillages dès la fouille et d'adapter les prélèvements malacofauniques aux types d'accumulations: dépotoirs alimentaires plus ou moins denses, déchets d'activités spécialisées, dépôts funéraires... Si à Beauvoir-sur-Mer le malacologue est intervenu en aval des opérations de fouille, il est désormais actif dès la fouille dans la région Pays de la Loire, comme au Langon, à Jard-sur-Mer en Vendée, à Piriac-sur-Mer et à Saint-MichelChef-Chef en Loire-Atlantique.

\section{Présentation du SITE DE BEAUVOIR-SUR-Mer}

L'extension connue de l'amas coquillier de Beauvoir-sur-Mer est de $1700 \mathrm{~m}$ de long pour une largeur observée de $25 \mathrm{~m}$ et une hauteur de 1 à 2 m (Fig. 1). Actuellement, il est localisé à environ $2 \mathrm{~km}$ du trait de côte, son extrémité présumée est localisée à environ $400 \mathrm{~m}$ de l'étier du Dain et $1,4 \mathrm{~km}$ du port du Bec (Fig. 2). Des argiles holocènes se sont préférentiellement déposées à l'extrémité de la péninsule de Beauvoir, du fait de convergences de courants venus de la Baie de Bourgneuf au nord et du Goulet de Fromentine au sud, d'une manière comparable à l'actuel passage du Gois qui relie l'île de Noirmoutier au continent à marée basse.

Des bassins versants opposés ont tracé des talwegs, dont la situation est perpendiculaire à la « rive». Transformés en étiers au contact du schorre, ces chenaux ont été entretenus très tôt vers la baie de Bourgneuf et effacés au sud vers le marais de Monts. Cette morphologie pourrait laisser entendre une relation entre l'allongement du dépôt d'huîtres et la place du «talliea portus de Belveario » relaté par le cartulaire de Coudrie (1180), ou bien un exhaussement du cordon vaseux.

\section{LA CHRONOLOGIE DE LA FRÉQUENTATION DU SITE \\ DE BEAUVOIR-SUR-MER PAR LES HOMMES}

La réalité archéologique nous montre que ce schorre était occupé par les Romains à la fin du IIe siècle, à $400 \mathrm{~m}$ de la côte rocheuse, à $2 \mathrm{~m} \mathrm{NGF}$ (nivellement général de la France) (Fig. 3). Un niveau de céramiques domestiques versées sur un plan était bordé d'une butte de 0,50 m de hauteur, datée du sub-boréal. Cette «bosse», qui aurait pu avoir une signification dans un ensemble salicole de type marais salants, a été disposée au détriment d'un sédiment profond, puisque les vases contenues au toit du substrat à cet emplacement (-0,60 m NGF) sont datées de $5580 \pm 380$ BP (Fig. 3). Huit siècles au moins ont séparé les premières jetées gallo-romaines des traces archéologiques du Moyen Âge. Finalement, pour vérifier l'hypothèse de la présence de marais salants galloromains, il suffirait de faire effectuer une fouille fine sur 3 ha du terrain par des géoarchéologues.

Une datation radiocarbone sur une des huîtres de l'amas coquillier avait déjà permis d'attribuer une partie de l'accumulation du dépôt entre les XIe et XIVe siècles. $(900 \pm 60$ BP, 5702 ; Ters \& Viaud 1983). L'imprécision de la calibration de cette datation est liée à l'effet réservoir des datations faites sur coquilles 


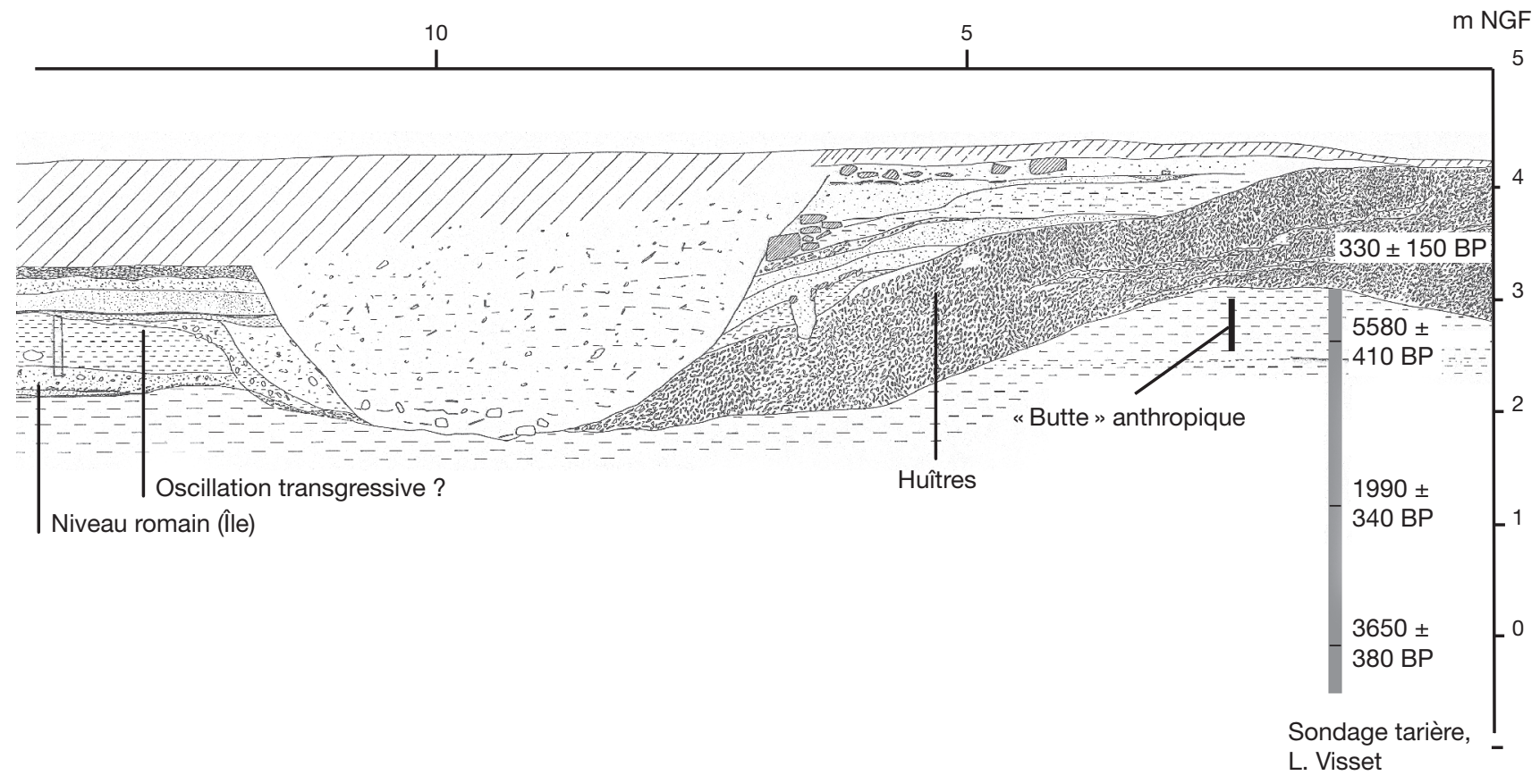

FIG. 3. - Coupe stratigraphique au travers du dépôt d'huîtres de Beauvoir-sur-Mer. Abréviation: NGF, nivellement général de la France (N. Rouzeau, SRA).

marines (delta R de $-260 \pm 65$ : Marchand et al. 2009; deux sigma: Stuiver \& Reimer 1993; Hughen et al. 2004). C'est à l'occasion d'un sauvetage d'urgence lié à la construction d'un lotissement en 1985, qu'une seconde date a été obtenue par accélérateur Lyon-3677 (Sac-6188) sur une coquille d'huître; elle indique $1315 \pm 30 \mathrm{BP}$, soit un âge calibré de 680 à 981 de notre ère, si on tient compte de l'effet réservoir. Ainsi, ces datations sur coquilles d'huîtres témoignent d'une formation de cet amas coquillier entre les VIIe et XIVe siècles.

Le second élément datant de ces accumulations est lié à la construction d'un château qui aurait été érigé sur le dépôt au XIIIe siècle (Soret 1994). Les sondages que nous avons menés montrent bien que le tas d'huîtres a été constitué avant la construction de ce château, mais qu'il s'est poursuivi tout le long de son occupation. Ainsi, la seule information apportée par ce second élément est l'exploitation de ces huîtres avant le XIII" siècle.

Bien que la datation de cette accumulation d'huîtres reste imprécise et que ce site soit isolé, en l'état actuel de nos connaissances de tout habitat contemporain, son analyse nous semblait primordiale dans le but de caractériser l'activité liée à ces coquilles. Seul l'intérêt scientifique porté à ce type de site permettra de motiver de nouvelles fouilles archéologiques sur des accumulations similaires et ainsi de découvrir à la fois des éléments datant et les occupations humaines qui ont pu y être associées.

\section{LES ÉTUDES MALACOLOGIQUES}

Bien que les huîtres de l'amas coquillier de Beauvoir-sur-Mer n'aient jamais été examinées par un malacologue avant les années 1980 (Gruet pers. comm.) puis en 2006, de nom- breuses mentions de ce site sont connues (Rivière 1834; Mourain de Sourdeval 1864; Baudoin 1912; Begouen 1937; Rousseau 1963; Soret 1985). Tous ces auteurs décrivent ce site à l'occasion de travaux d'aménagement. Leur attention avait été attirée par sa composition quasi-exclusive d'huîtres.

\section{LES AUTRES ACCUMULATIONS D'HUÎTRES DU LITTORAL ATLANTIQUE FRANÇAIS}

L'accumulation de Beauvoir-sur-Mer n'est pas la seule du littoral atlantique français qui soit composée quasi exclusivement d'huîtres plates avec des individus jointifs et localisée à la limite d'un ancien marais aujourd'hui asséché: Bourgneufen-Retz (Loire-Atlantique), Champagné-les-Marais (Vendée), Le Langon et Saint-Michel-en-L'Herm (Vendée) présentent les mêmes caractéristiques (Fig. 2; Baudoin 1912; Rousseau 1963 ; Verger 1968; Soret 1985; Gruet \& Prigent 1986 ; Verger 2005; Dupont \& Bougeant 2008). Beaucoup n'ont pas été étudiés dans leur intégralité. Ainsi, leurs volumes et datation ne sont pas toujours connus avec exactitude.

Celui de Saint-Michel-en-L'Herm est le mieux connu, car il a été exploité entre 1924 et 1964 pour amender les sols et pour construire des routes. Son volume a été évalué entre 200000 et $600000 \mathrm{~m}^{3}$. Cet amas coquillier, comme à Beauvoir-surMer, est placé sur le schorre holocène, une vase appelée bri (Verger 2005). La position stratigraphique de Bourgneufen-Retz est connue grâce à un curage de fossé. Sa position au-dessus d'un sédiment vaseux flandrien est l'élément qui a incité les auteurs à attribuer sa formation à la même période que ceux de Beauvoir-sur-Mer et de Saint-Michel-en-L'Herm. 
Celui de Champagné-les-Marais est connu uniquement par prospection et il n'existe pas, à notre connaissance, d'informations concernant la chronologie de sa formation.

Des huîtres ont aussi été identifiées dans plusieurs points du village du Langon lors des prospections menées par E. Bernard, J. Pascal et N. Rouzeau (Bernard 1994) dans l'environnement de cet important vicus installé au cour des salines gauloises (Rouzeau et al. 2002). La situation d'un "dépôt de cendres", qui correspond au rebut de la fabrication ignigène du sel du Langon, se rapproche des observations portées plus haut sur les niveaux marins à l'époque romaine. Car, là, ce ne sont pas les huîtres qui font la chaussée, mais le dépôt de cendres sondé par Émile Bernard, archéologue départemental, sur $400 \mathrm{~m}$ de longueur et $30 \mathrm{~m}$ environ de largueur, disposé le long du Quai du Port. De la même façon, nous avons trouvé à Beauvoir, dans le sondage effectué sous le château, des débris de fours à sel pré-augustéens de type Vénète. Le Quai du Port du Langon est actuellement à $23 \mathrm{~km}$ par voie d'eau de l'accumulation d'huîtres des Chaux, de Saint-Michel-enL'Herm, bourg maintenant éloigné de la mer de $8 \mathrm{~km}$. Il serait intéressant de savoir si ces sites ont pu être reliés les uns aux autres par voies de navigation, ou si les distances entre les sites d'exploitation d'huîtres et la mer ne sont pas liées à des chronologies différentes d'occupation. La distance au trait de côte, plus importante au Langon qu'à Saint-Michel-enL'Herm, pourrait ainsi refléter l'évolution du trait de côte, qui recule sur la mer du fait du taux de sédimentation important sur cette partie du littoral.

Notre connaissance sur le type d'exploitation des huîtres réalisée au Langon a été complétée en 2008 par notre présence sur la fouille (Dupont \& Bougeant 2008). Bien que très localisée, cette fouille dirigée en 2007 par M. L. Hervé de l'INRAP a permis de confirmer la formation des accumulations d'huîtres du Langon à l'époque romaine. Les points communs de ce dépôt coquillier avec celui de Beauvoir-sur-Mer sont sa possible localisation à la marge d'un ancien marais, sa composition quasi-exclusive d'huîtres et la présence d'huîtres trouvées valves jointes. Ces dépôts ne semblent pas liés à la consommation régulière d'une population gallo-romaine. En effet, contrairement aux dépotoirs domestiques, ces dépôts sont composés quasi-exclusivement d'huîtres. La fouille n'a pas montré de structures d'habitats et les structures anthropiques observées témoignent toutes d'activités tournées vers l'artisanat.

Il existe d'autres références à des accumulations d'huîtres beaucoup plus récentes en France, comme celles de Granville (Manche) et de Cancale (Ille-et-Vilaine) qui ont été édifiées jusqu'au XVIIIe siècle. Par exemple, celles de Granville faisaient approximativement $300 \mathrm{~m}$ de long sur $200 \mathrm{~m}$ de large, avec une hauteur de 2 à $3 \mathrm{~m}$ en 1830 (Levesque 1982).

\section{INTERPRÉTER LES DÉPÔTS D'HUÎTRES : LES NOUVELLES APPROCHES}

Par le passé, plusieurs de ces dépôts d'huîtres ont été attribués à des accumulations naturelles du fait de la présence d'huîtres jointives au sein même de l'accumulation. Cependant, cette observation d'huîtres complètes n'est pas incompatible avec l'ouverture de ces huîtres et l'origine anthropique de ces accumulations.

À partir de l'analyse des coquillages de Beauvoir-sur-Mer, notre premier objectif est de comprendre comment ces hû̂tres ont été exploitées. Dans ce but, les stigmates liés d'une part aux faunes marines associées et d'autre part aux outils utilisés par les hommes ont été recensés. La biométrie a aussi été réalisée afin de savoir si les huîtres ont été sélectionnées lors de leur collecte et si toutes les huîtres transportées sur Beauvoir-sur-Mer ont été ouvertes. D'autres paramètres, comme la dimension des huîtres ouvertes et la proportion des valves jointes, apportent des informations sur le degré de spécialisation de ce lieu d'exploitation d'huîtres.

\section{LES CONDITIONS DE FOUILLES DE BEAUVOIR-SUR-MeR}

Lors de notre intervention, la partie du site de Beauvoir-surMer explorée en 1985 venait de faire l'objet d'un décapage général et les voiries étaient déjà posées. Les questions liées aux huîtres apparaissaient à beaucoup comme secondaires en raison de la présence à proximité d'un château médiéval. Ainsi, l'autorisation de fouiller en sauvetage programmé a été remplacée par une autorisation de sondage. Nous avons dégagé des fondations de construction, faites pour moitié de galets de délestage associés à un niveau de sol recouvert d'ardoises épaisses et percées. C'est après nous être assurés que les constructions étaient postérieures au niveau d'huîtres que N. Rouzeau a prélevé un échantillon intact de $0,32 \mathrm{~m}^{3}$. L'étude qui suit a été réalisée sur ce prélèvement de l'amas coquillier de Beauvoir-sur-Mer. Il faut noter que l'emplacement de ce sondage correspond à la localisation des annexes du château qui ont fonctionné postérieurement aux accumulations d'huîtres. Le sondage du château montre que les huîtres étaient déversées de manière à consolider le haut du talus, plus qu'à combler des fossés (Fig. 3). Une coupe stratigraphique axiale a aussi été réalisée à l'Ouche sur $25 \mathrm{~m}$ de largueur et $400 \mathrm{~m}$ de long à l'ouest de ces sondages (Fig. 1). C'est sur un échantillon de coquilles de cette coupe qu'Yves Gruet a réalisé une étude préliminaire restée inédite.

\section{ANALYSE MALACOLOGIQUE: MATÉRIEL ET MÉTHODE}

L'analyse des coquilles qui va suivre est quant à elle issue du bloc d'huîtres prélevé lors de la fouille «Beauvoir Le Château, $n^{\circ}$ 85-018-001». Il a été emporté intact dans du plâtre afin d'être fouillé huître après huître en laboratoire. Les huîtres ont ensuite été extraites une à une à la main. Elles ont été conditionnées dans des caisses sur des feuilles de papier journal sans être lavées. Les exemplaires entiers ont été conservés valves jointes. En 2006, ces huîtres ainsi conditionnées ont été étudiées par C. Dupont. Pour ce faire, elles ont été examinées avant et après lavage. Ce dernier a été réalisé à l'eau douce sur une colonne de tamis de mailles de 2 et $0,5 \mathrm{~mm}$ pour recueillir tous les fragments de coquilles et autres indices associés aux tests d'huîtres.

La position de l'empreinte musculaire a permis de latéraliser les valves d'huîtres. Tous les fragments d'huîtres qui n'ont pas pu être latéralisés ont quant à eux été décomptés lorsque leur 


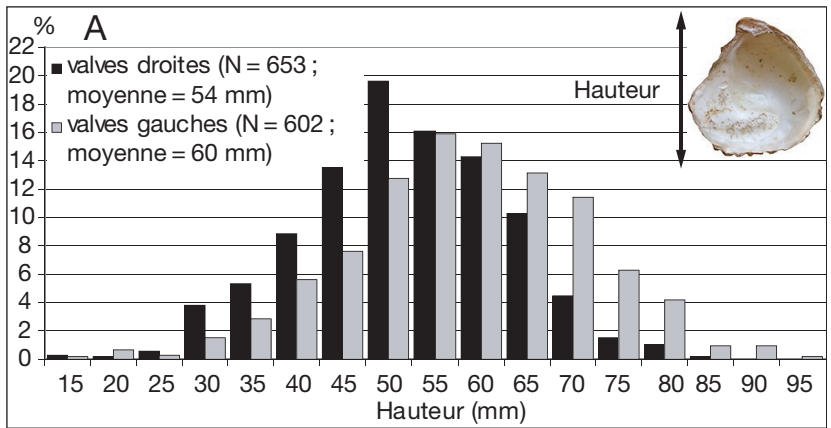

$\%$ B
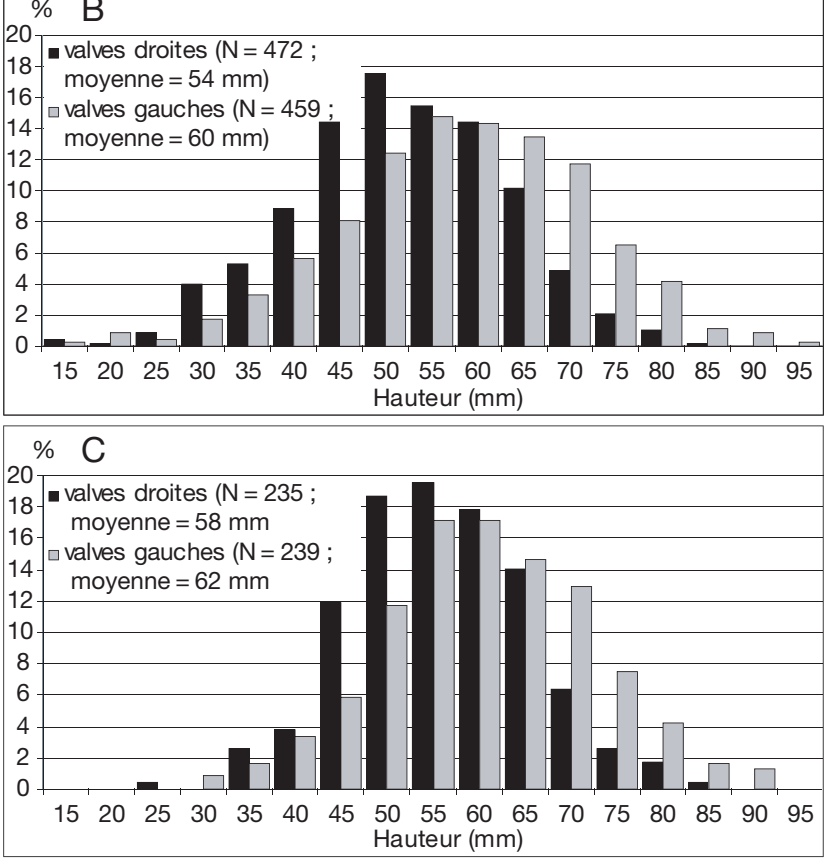

FIG. 4. - Histogramme de distribution de la hauteur des huîtres de Beauvoirsur-Mer: A, toutes huîtres comprises; B, huîtres jointes; C, huîtres avec stigmates d'ouverture. Abréviation: N, nombre valves étudiées (C. Dupont, CNRS).

dimension était supérieure à $5 \mathrm{~mm}$. Au total, 5673 restes de coquilles ont été comptés, pour 1919 valves droites et 1814 valves gauches. Ainsi, le nombre minimum d'individus (NMI) de l'échantillon prélevé est de 1919.

\section{LA COLLECTE DES HUÎTRES}

\section{L'ABSENCE DE CALIBRAGE DES HUÎTRES COLLECTÉES}

La distribution de la hauteur de toutes les valves droites et gauches échantillonnées dans le dépôt archéologique montre que toutes les dimensions sont représentées : petites et grandes ont été transportées sur le site (Fig. 4A).

La présence de valves jointes peut être liée au transport d'huîtres vivantes sur le site de Beauvoir-sur-Mer. Si seules les mesures des huîtres trouvées valves jointes sont considérées, (Fig. 4B) la hauteur moyenne des valves droites est de $54 \mathrm{~mm}$ pour $60 \mathrm{~mm}$ pour les valves gauches. Ces valeurs sont les mêmes que celles observées pour l'ensemble des huîtres. Ainsi petites et grandes huîtres ont été transportées vivantes sur le site de Beauvoir-sur-Mer.
TABLEAU 1. - Pourcentage des stigmates associés à l'épifaune et à l'endofaune observés sur les huitres du dépôt archéologique de Beauvoir-sur-Mer. Abréviation: $\mathbf{N}$, nombre de valves étudiées.

\begin{tabular}{lrr}
\hline Epifaune et endofaune & $\begin{array}{r}\text { Valves droites } \\
\text { (N=848) }\end{array}$ & $\begin{array}{r}\text { Valves gauches } \\
\text { (N=823) }\end{array}$ \\
\hline Absence & $63,6 \%$ & $24,7 \%$ \\
Serpulidés & $3,8 \%$ & $9,7 \%$ \\
Eponge marine (Cliona) & $0,4 \%$ & $6,2 \%$ \\
Polydora sp. & $8,8 \%$ & $12,9 \%$ \\
Bryozoaires & $1,8 \%$ & $4,6 \%$ \\
Ocenebra sp. & $7,4 \%$ & $5,6 \%$ \\
Balanes & $0,1 \%$ & $0,0 \%$ \\
Spirorbis sp. & $0,2 \%$ & $0,5 \%$ \\
Naissain & $1,2 \%$ & $3,9 \%$ \\
Huîtres vivantes & $4,3 \%$ & $26,9 \%$ \\
Association avec des huîtres & $0,8 \%$ & $12,4 \%$ \\
mortes & $1,3 \%$ & $15,4 \%$ \\
Ancienne fixation à des huîtres & & \\
\hline
\end{tabular}

\section{LA FAUNE MARINE ASSOCIÉE AUX HUITTRES:} UNE IMAGE DE L'ENVIRONNEMENT EXPLOITÉ

Pour comprendre comment ces huîtres ont été collectées, il semble important de connaître les caractéristiques de l'environnement dans lequel elles se sont développées. Les stigmates dus à l'endofaune et à l'épifaune associées aux huîtres nous en donnent une image indirecte. Certaines des huîtres étaient encore agrégées à d'autres individus d'huîtres dans le dépôt archéologique (Tableau 1). Quand aucun indice ne témoignait de leur collecte à l'état mort, comme des stigmates d'organismes marins à l'intérieur de la valve ou un aspect poli du contour des valves, ces valves d'huîtres ont été considérées comme des "huîtres vivantes" au moment de la collecte. Les coquilles d'huîtres qui présentent des stigmates liés à l'épifaune et à l'endofaune sur la face interne des valves étaient déjà "mortes" au moment de la collecte. Un grand nombre d'huîtres étudiées ont des indices d'ancienne fixation à d'autres huîtres: face plane et présence de fragments de coquilles d'huîtres sur la face externe. Ces huîtres anciennement associées ont pu être séparées pendant leur collecte, mais aussi après leur rejet par l'action de multiples agents taphonomiques. Si tous ces indices d'association d'huîtres sont pris en compte (Tableau 1), plus de $51 \%$ des huîtres exploitées étaient attachées à une autre huître lors de leur collecte. Ainsi, ce bivalve a sans doute été exploité dans un banc dense en huîtres, de type huîtrière naturelle.

D'autres stigmates liés à la faune marine associée aux huîtres ont été observés (Tableau 1). Les occurrences de ces faunes associées sont plus nombreuses sur les valves gauches que sur les valves droites à Beauvoir-sur-Mer. Cette observation est régulièrement faite sur les sites archéologiques (Dupont pers. comm.). Les huîtres se fixent à leur support par la valve gauche ou valve creuse. La droite est quant à elle mobile et est directement soumise aux courants marins. L'espace créé entre la valve gauche et le substrat peut procurer un environnement plus abrité pour les faunes marines associées. 
Les décomptes du Tableau 1 correspondent uniquement aux coquilles les mieux conservées. Ils montrent que quelques huîtres ( $6 \%$ des valves gauches) ont été attaquées par l'éponge marine Cliona Grant, 1826 inféodée à la zone subtidale. Ce taux est insuffisant pour témoigner d'un dragage de l'ensemble des huîtres dans la zone subtidale. Il montre que certaines huîtres proviennent certainement des zones les plus basses de l'estran et ont pu être exploitées lors de grands coefficients de marée. La fréquence du Polydora Bosc, 1802, un ver inféodé aux environnements envasés, est de $13 \%$ sur les valves gauches. Ce pourcentage n'est pas assez important pour prouver une exploitation dans des fonds envasés, mais montre que la zone exploitée était sans doute abritée des houles dominantes. Un tel environnement peut correspondre à ce qui est actuellement présent dans les zones moyennes des estrans de la baie de Bourgneuf. L'hypothèse d'une zone largement envasée n'est pas confirmée par les restes coquilliers des autres espèces, trouvés dans le sédiment associé à ces huîtres: Patella sp., Mytilus edulis Linnaeus, 1758, Littorina littorea (Linnaeus, 1758), Gibbula umbilicalis (da Costa, 1778), Cerastoderma edule (Linnaeus, 1758), Mimachlamys varia (Linnaeus, 1758) et Chiton sp. Ces différentes espèces sont au contraire inféodées à un environnement rocheux et Patella sp., Gibbula umbilicalis plus strictement à la zone intertidale. Ces espèces peuvent correspondre à la présence de roches dans la proximité de la zone exploitée pour les huîtres. Des stigmates liés au murex Ocenebra sp., observés sur les huîtres (Tableau 1), montrent que la zone exploitée n'était pas influencée par les eaux douces.

Enfin, une balane, sans doute une Balanus crenatus Bruguière, 1789, identifiée par Y. Gruet, a été trouvée sur une valve droite d'huître. Le sédiment associé aux huîtres ayant été tamisé, la rareté des balanes ne peut pas être due à leur détachement des tests d'huîtres à leur mort ou lors de leur enfouissement dans le sédiment. De plus, lorsqu'elles sont abondantes dans l'environnement des huîtres, elles se retrouvent en partie fixées à la surface de ce bivalve, comme par exemple à Saint-Michel-en-L'Herm (Baudoin 1916a, b; Gruet \& Prigent 1986). La rareté des balanes sur les huîtres de Beauvoir-sur-Mer peut correspondre à un environnement qui n'a pas permis le développement de ce crustacé, comme par exemple une densité extrêmement importante en huîtres.

\section{LES OUTILS UTILISÉS POUR EXPLOITER LES HUITTRES}

Des perforations observées sur les tests d'huîtres ont attiré notre attention (Fig. 5). Les huîtres ayant été ramassées à la main lors du démantèlement du niveau coquillier, ces perforations ne peuvent pas être liées à l'utilisation d'outils de fouille (truelle, piochon). De plus, ces perforations étaient couvertes de sédiment, ce qui plaide en faveur de leur origine ancienne. Au total, 20 valves percées ont été observées. En dépit de leur nombre réduit comparé aux 3724 valves d'huîtres observées, ces stigmates sont peut-être liés aux outils utilisés pour exploiter ces huîtres.

Parmi les 20 valves, 19 sont des droites et une seule est une valve gauche. Cette dernière était associée à une valve droite percée (Fig. 5 F). Cet individu d'huître dont les deux valves ont été percées semble correspondre à l'action d'un outil qui a transpercé toute l'épaisseur de l'huître. La prédominance de perforations sur les valves droites renforce leur possible formation pendant leur collecte. En effet, comme nous l'avons mentionné plus haut, les naissains d'huîtres se fixent à leur support par leur valve gauche, par un ciment, en épousant la forme de leur support ou substrat. La valve droite est quant à elle plus vulnérable face à l'action d'un outil.

Dans le but d'avoir des informations supplémentaires sur les types d'outils utilisés, ces perforations ont été analysées en fonction de leur forme et de leur dimension (Fig. 6). L'intervalle de confiance permet, en dépit du faible nombre de valeurs mesurées, de prendre en compte l'ensemble de la variabilité des dimensions des perforations pour les différentes formes observées. Les valeurs des intervalles de confiance sont proches des valeurs maximales et minimales mesurées, du fait du faible nombre d'échantillons. Ainsi, les perforations les plus arrondies semblent plus petites, alors que les plus grandes semblent plus anguleuses. Ces différences pourraient correspondre à des degrés d'enfoncement différents d'un outil. L'extrémité des dents de cet outil pourrait être arrondie tandis que sa base pourrait être anguleuse.

Cette hypothèse de degrés d'enfoncement différents d'un même outil semble contredite par la présence d'impacts, les uns de forme arrondie, les autres de forme anguleuse (Fig. $5 \mathrm{G}, \mathrm{H}$ ). Il est donc possible que plusieurs outils aient été utilisés pour exploiter ces huîtres: les uns plus fins aux extrémités arrondies, les autres aux dents plus larges et de forme anguleuse.

Plusieurs outils sont connus dans l'exploitation des huîtres dont la drague, le râteau, le système de doubles râteaux ou griffe et le piochon (Fig. 7; Duhamel du Monceau 1769; Clerc 1828; Grelon 1978; George \& Nédélec 1991). La drague et longs râteaux peuvent être utilisés à bord d'un bateau (Guillet \& Guillet 2008). Le piochon et le râteau à long manche sont utilisés pour exploiter la zone intertidale accessible à pied sec. Jacques \& Ronan Guillet (2008) reprennent un dessin de L'Illustration du 30 janvier 1892 , où hommes et femmes collectent des huîtres à l'aide de râteaux à petits et grands manches. Sur ce dessin, on distingue une collecte sélective des plus grandes huîtres. Louis Lacroix (1942) rapporte l'existence d'un compte rendu du 8 avril 1777 où, dans certaines zones de la côte atlantique, les huîtres étaient si denses qu'elles ne pouvaient être draguées, mais enlevées par blocs à coups de pioche.

La basse fréquence des perforations observées pourrait correspondre à un mouvement de ratissage des fonds marins à l'aide de râteaux, plutôt que de piochons, qui sont plus destructifs pour les huîtres et auraient conduit à un taux de perforation plus important. L'hypothèse de l'utilisation d'une pioche pour prélever des blocs d'huîtres ne peut pas non plus être exclue.

\section{LES ACTIVITÉS LIÉES À L'EXPLOITATION DE LA CHAIR DES HUÎTRES}

\section{NOUVEAU REGARD SUR LES STIGMATES D'OUVERTURE}

Afin de quantifier les stigmates d'ouverture observés, ceux-ci ont été classés en différents types, qui correspondent à ceux qui sont régulièrement rencontrés en contextes archéologiques (Fig. 8). Ils ont atteint $59 \%$ des valves droites et $67 \%$ des valves gauches. L'encoche de plus de $5 \mathrm{~mm}$ de large (Fig. 8 stigmate 4) est le 

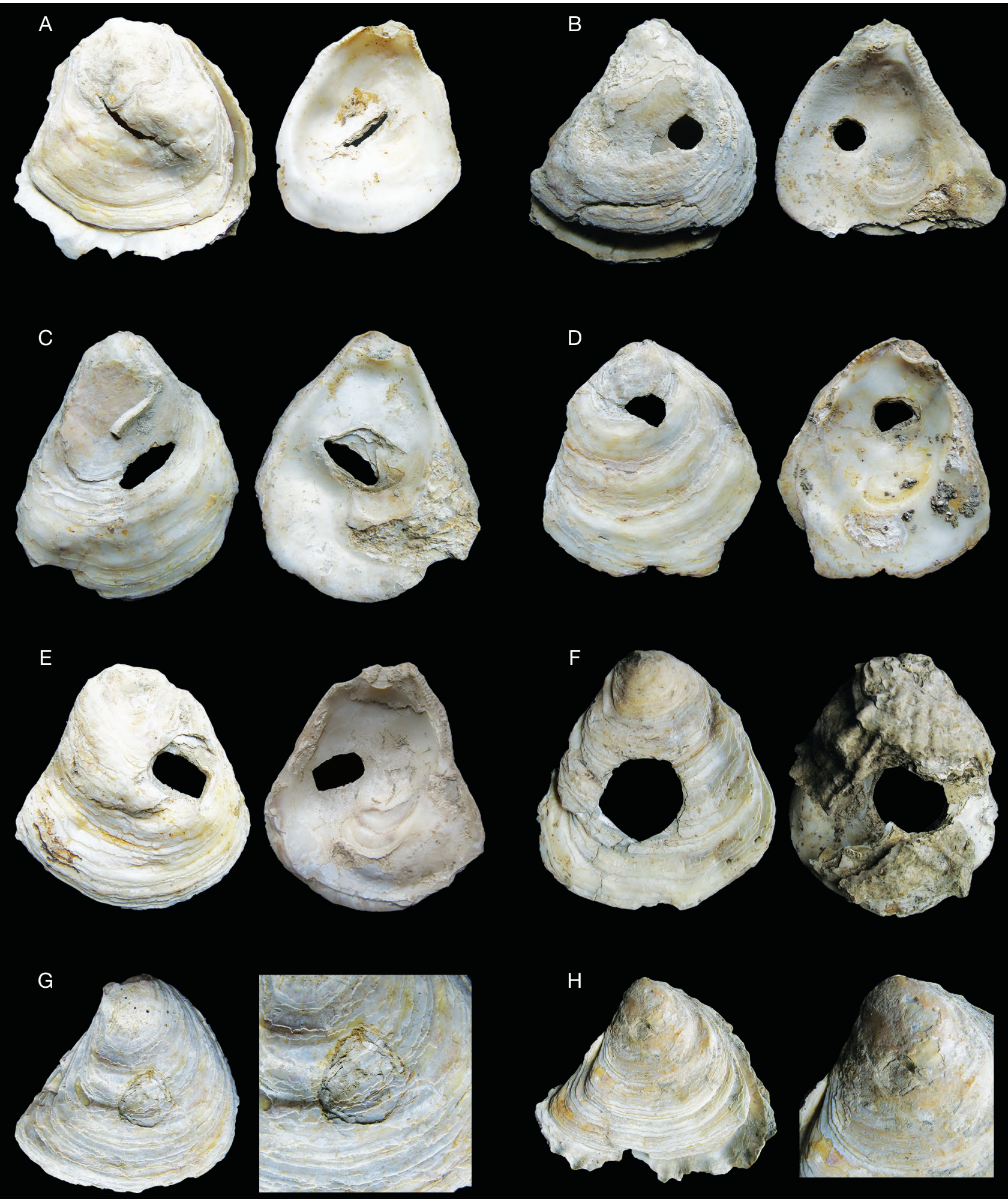

FIG. 5. - Différentes formes de perforation observées sur les huîtres de Beauvoir-sur-Mer: $\mathbf{A}$, forme de fente $(L=77 \mathrm{~mm}) ; \mathbf{B}$, forme circulaire ( $\mathrm{L}=85 \mathrm{~mm})$; C forme ovale $(\mathrm{L}=98 \mathrm{~mm})$; $\mathbf{D}$, forme triangulaire $(\mathrm{L}=84 \mathrm{~mm})$; $\mathbf{E}$, forme quadrangulaire $(\mathrm{L}=91 \mathrm{~mm})$; $\mathbf{F}$, forme pentagonale $(\mathrm{L}=111 \mathrm{~mm})$. Impacts de l'outil sur la surface de I'huître: $\mathbf{G}$, impact pentagonal $(\mathrm{L}=90 \mathrm{~mm})$; $\mathbf{H}$, impact circulaire $(\mathrm{L}=120 \mathrm{~mm})$ (C. Dupont, $C N R S)$. 


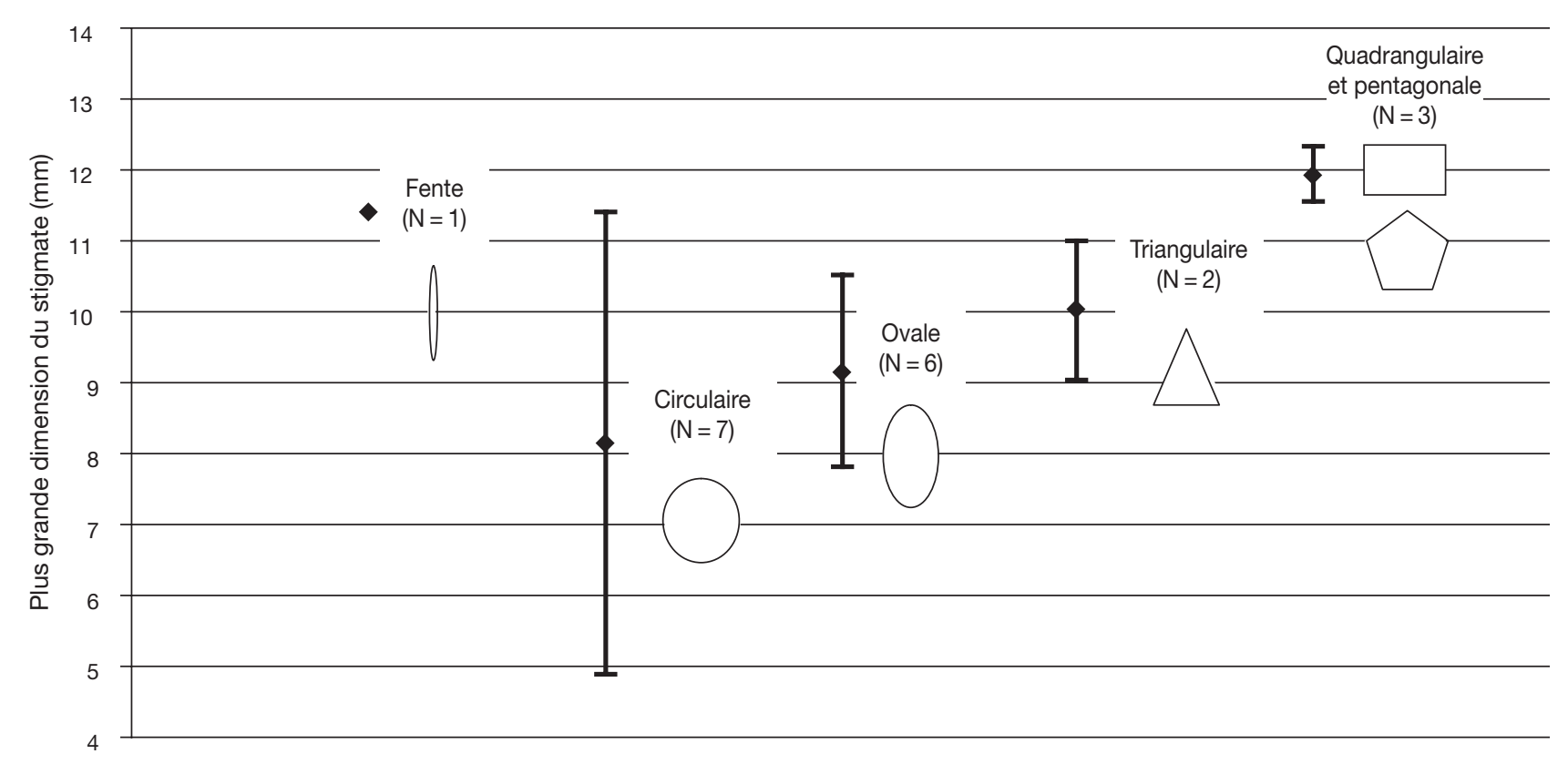

FIG. 6. - Mesure de la plus grande dimension des perforations en fonction de la forme du stigmate. Abréviation: N, nombre de valves étudiées (C. Dupont, CNRS).
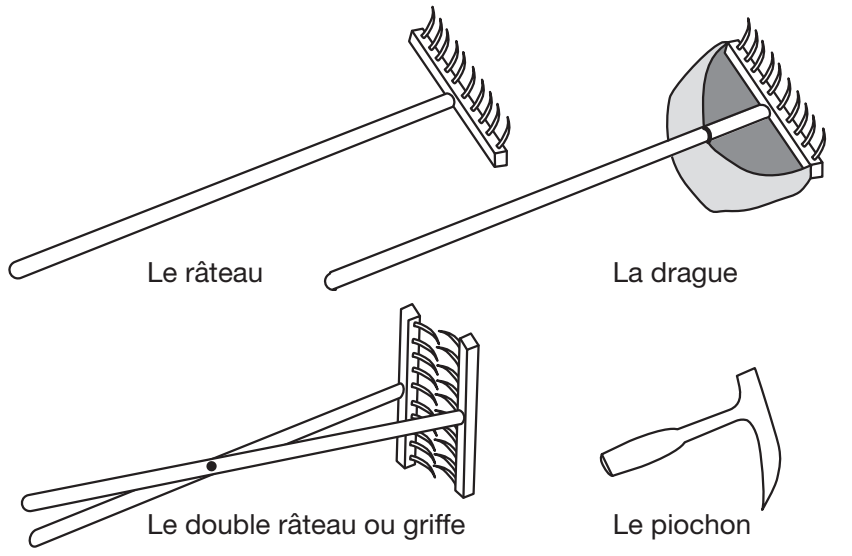

FIG. 7. - Exemples d'outils potentiellement utilisés pour exploiter les huîtres (C. Dupont \& L. Quesnel, CNRS).

stigmate le plus récurrent (Fig. 9). La largeur moyenne de ce stigmate est de $8 \mathrm{~mm}$, ce qui peut correspondre à la largeur de la lame du couteau utilisée pour ouvrir les huîtres. Le mouvement d'ouverture des huîtres semble standardisé. Les stigmates d'ouverture sont localisés sur le bord coquillier opposé à la charnière (Fig. 10). C'est d'ailleurs cette localisation (Baudoin 1916c; Begouen 1937) qui fait que Gambier \& Robuchon (1936) n'avaient pas, à l'époque, identifié les stigmates d'ouverture sur les buttes de Saint-Michel-en-l'Herm. Pour ces deux auteurs, l'ouverture des huîtres devait se faire au niveau de la charnière du coquillage. N'ayant pas observé de séquelles sur cette zone, les auteurs avaient donc conclu à l'origine naturelle du dépôt. En fait, les deux pratiques ont été décrites. Verger témoigne en 1959 d'un exemple très démonstratif. En effet, les écaillères de Granville ouvrent les huîtres dans le sens contraire des écaillères de Paris, qui les ouvrent par la charnière. La variabilité de la forme peut difficilement expliquer cette différence, car une partie des huîtres transportées fraîches et ouvertes à Paris provenaient de Granville.

Nous avons montré précédemment que des huîtres de toutes tailles ont été collectées: petites et grandes. Mais ont-elles toutes été ouvertes? L'histogramme $\mathrm{C}$ de la Figure 4 correspond à la distribution des hauteurs des huîtres qui présentent des stigmates d'ouverture. Comparé à l'ensemble des huîtres trouvées sur le site (Fig. 4 A), ce graphique, qui exclut les huîtres sans stigmate, témoigne de dimensions plus grandes avec $58 \mathrm{~mm}$ de moyenne pour les valves droites et $62 \mathrm{~mm}$ pour les valves gauches. Cette différence entre les graphiques montre que les petites huîtres ont été collectées sur estran, puis transportées à Beauvoir-sur-Mer. Mais une fois sur le site, ces huîtres ont été abandonnées sans être ouvertes. Ces petits individus ont été acheminés sur le site, fixés à de grands individus d'huîtres. Certains petits exemplaires étaient encore fixés aux grandes huîtres lors de leur analyse.

\section{LES HUÎTRES JOINTIVES}

Vingt-huit pour cent du NMI des huîtres de Beauvoir-surMer étaient jointives lors de l'étude. Ce pourcentage est sans doute sous-estimé, du fait d'une dislocation possible de certains individus jointifs lors de processus taphonomiques, mais aussi de leur transport après la fouille. La présence d'huîtres jointives n'est pas incompatible avec leur consommation. En effet, certains individus jointifs présentent des stigmates d'ouverture sur leur face interne (Fig. 11).

$\mathrm{Si}$, comme en témoignent les nombreux stigmates d'ouverture, ces huîtres de Beauvoir-sur-Mer ont bien été ouvertes, pourquoi les trouve-t-on jointives et refermées dans le dépôt? 

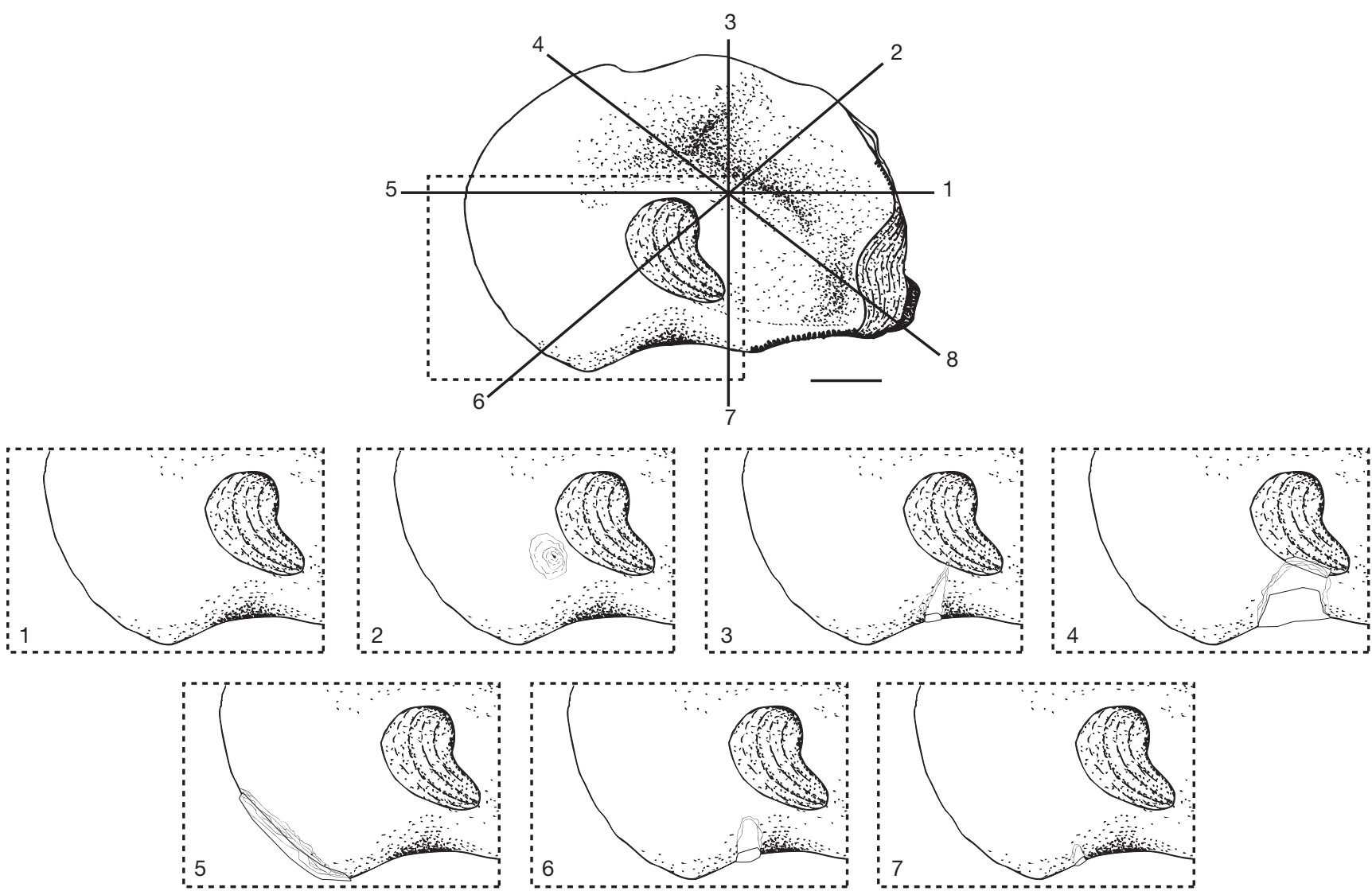

FIG. 8. - Typologie des stigmates liés à l'ouverture des huîtres: 1, absence de stigmate; 2 , impact sous forme d'esquille circulaire ou allongé sans cassure du bord coquillier; $\mathbf{3}$, échancrure pointue; $\mathbf{4}$, encoche de largeur égale ou supérieure à $5 \mathrm{~mm} ; \mathbf{5}$, cassure rectiligne du bord coquillier; $\mathbf{6}$, encoche de largeur inférieure à $5 \mathrm{~mm}$ plus profonde que large; 7 , encoche de largeur inférieure à $5 \mathrm{~mm}$ plus large que profonde (l'orientation des stigmates est arbitraire sur cette figure). Échelle: $1 \mathrm{~cm}$ (C. Dupont \& L. Quesnel, CNRS).

Deux éléments ont favorisé la préservation des valves jointes. Le premier est le muscle adducteur, et le second, le ligament. Louverture des huîtres nécessite la section du muscle. Les valves d'huîtres peuvent alors être écartées et la chair extraite sans disloquer les deux valves. Le ligament localisé à la charnière des valves maintient les huîtres entrouvertes lors de leur abandon. Sous le poids du sédiment et des autres huîtres, elles se referment. Le ligament est difficile à observer en contexte archéologique, car il se détache des valves lors de leur lavage. Quelques-uns ont été observés à Beauvoir-sur-Mer. La façon dont les huîtres ont été consommées ou préparées n’a pas nécessité la séparation des deux valves.

\section{COMPARAISON DES ACCUMULATIONS \\ D'HUÎTRES DE BEAUVOIR-SUR-MER \\ ET DE SAINT-MICHEL-EN-L'HERM}

Des similarités existent entre le site archéologique de Beauvoirsur-Mer et celui, en partie contemporain, de Saint-Michelen-L’Herm.

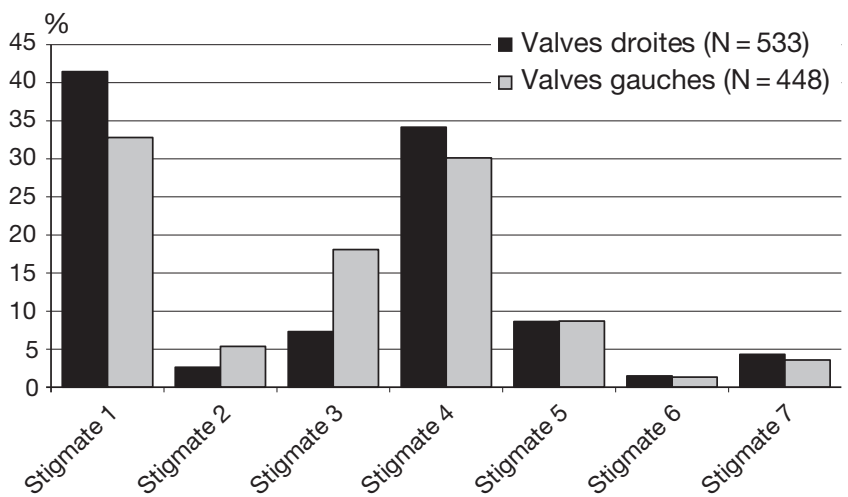

FIG. 9. - Distribution des stigmates d'ouverture d'huîtres observés sur les huîtres de Beauvoir-sur-Mer. Abréviation: N, nombre de valves étudiées (C. Dupont, CNRS).

Tous deux sont des accumulations médiévales, au moins en partie, d'huîtres déposées sur des vases flandriennes (Ters \& Viaud 1983). Ces dépôts coquilliers sont composés majoritairement d'huîtres. Cette caractéristique contraste avec la 


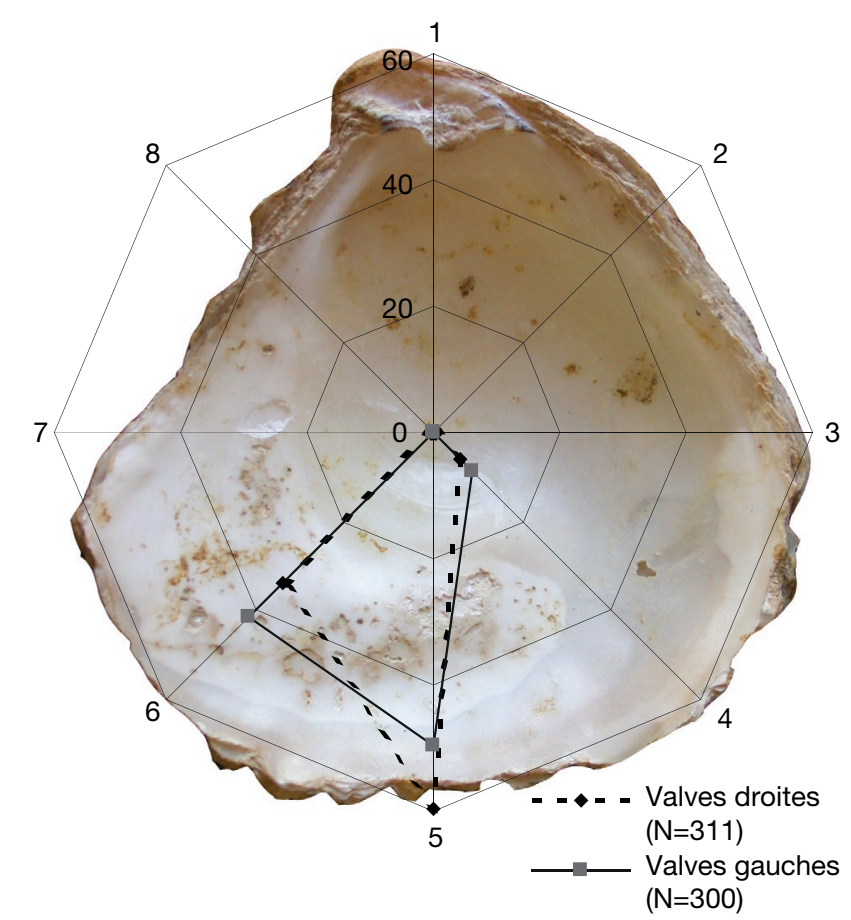

FIG. 10. - Localisation des stigmates d'ouverture observés à Beauvoir-sur-Mer Abréviation: N, nombre de valves étudiées (C. Dupont, CNRS).

diversité des coquillages trouvés dans des déchets alimentaires d'habitats côtiers et va dans le sens d'une activité spécialisée et centrée sur une espèce seulement.

La collecte des huîtres a sans doute été réalisée par ratissage d'une huîtrière naturelle sur estran, parfois lors de basses mers de vive eau. A Beauvoir-sur-Mer et à Saint-Michel-en-L'Herm, les pêcheurs n'ont pas pris le temps de séparer les petites huîtres des grands individus lors de la collecte (Baudoin 1916a, b; Gruet \& Prigent 1986). Aucun tri des coquillages n'a été fait avant l'ouverture des huîtres.

Dans ces deux dépôts coquilliers, certaines huîtres ont été trouvées jointives. Leur proportion à Beauvoir-sur-Mer $(28 \%$ du NMI) est similaire à ce qui est observé à Saint-Michel-enL'Herm (22\% à $40 \%$ du NMI pour deux échantillons étudiés; Gruet \& Prigent 1986). Le ligament qui garde les deux valves jointes a été observé sur plusieurs huîtres de Beauvoir-sur-Mer et de Saint-Michel-en-L'Herm (Quatrefages cité dans Baudoin 1916a, b; Gruet \& Prigent 1986). Plus de 59\% des huîtres de Beauvoir-sur-Mer (62\% à Saint-Michel-en-L'Herm; Gruet \& Prigent 1986) possèdent des stigmates liés à leur ouverture sur le bord coquillier opposé à la charnière. Ces observations attestent d'une extraction rapide de la chair de l'animal sans dislocation systématique des deux valves d'huîtres.

Mais qu'est devenue la chair ? Levesque (1982) note qu'en 1786, la chair des plus grandes huîtres pouvaient être transportées de novembre à la mi-avril, sans préparation, dans des paniers de paille. Le même procédé est décrit en 1873 par le docteur anglais Lister (cité dans Levasseur 2005). En dehors de cette période, l'huître pouvait être salée. Lister rapporte que dans du sel, la chair peut se conserver plus d'un mois. Ces huîtres «à potage» étaient destinées à être cuisinées et mises dans des ragoûts. Des textes datant du XVIIIe siècle, comme ceux que rapporte Le Masson du Parc en 1730, attestent de ces modes de transport des huîtres dans des paniers, soit fraîches durant l'hiver, soit marinées aux autres saisons, à Cancale et Granville. Le salage des huîtres ne peut pas être exclu à Beauvoir-sur-Mer, car le sel, ce produit marin de haute valeur, a été exploité et exporté à proximité de cette ville (Villalobos \& Ménanteau 2006).

Baudoin (1916a, b) décrit la forme serpentiforme des dépôts coquilliers de Saint-Michel-en-L'Herm. Elle pourrait être liée au dépôt de coquilles d'huîtres le long d'un ancien étier. Pour Beauvoir-sur-Mer, à défaut de vérifier l'authenticité d'éventuels passages d'eau pour le transport des huîtres par bateaux ou barques de l'estran vers les sites d'exploitation, nous restons prudents.

Le mode d'exploitation qui résulta des accumulations de Beauvoir-sur-Mer et de Saint-Michel-en-L'Herm apparaît particulièrement destructeur pour les huîtrières. Du fait du prélèvement massif de ces huîtres dans leur environnement, les petits individus sont prélevés avant d'avoir une taille satisfaisante pour la consommation et beaucoup n'ont pas été ouverts. Elles n'ont pas toutes pu se reproduire avant leur collecte. De plus, le fait de prélever des coquilles vides d'huîtres mortes limite les surfaces de fixation disponibles dans le milieu naturel pour les naissains. Ainsi, l'exploitation d'huîtres de Beauvoir-sur-Mer et de Saint-Michel-en-L'Herm a pu avoir un impact considérable sur la régénération des huîtrières exploitées et réduire la viabilité de cette activité à long terme. Aujourd'hui, ces bancs ont été épuisés. Quelquesuns d'entre eux étaient encore exploités pendant le XIXe siècle, mais étaient en danger de surexploitation (Cavoleau 1844; Lacroix 1942). Le Règlement sur la police de la pêche maritime côtière demandait aux pêcheurs d'huîtres de trier les huîtres et de remettre à l'eau " toutes les huîtres qui n'atteignaient pas les dimensions réglementaires, ainsi que les poussiers, sables, graviers et fragments d'écailles.» (Ducos 1853).

\section{CONCLUSION}

Le potentiel d'informations de ce genre de site archéologique ne doit pas être méconnu. Le gisement de Beauvoir est peutêtre loin d'être un épiphénomène. Il peut représenter un maillon d'un système d'exploitation complexe qui approvisionnait les villes en produits de la mer. En effet, plusieurs sites archéologiques reflétant le même type d'activité sont connus mais n'ont pas été étudiés. L'étude des huîtres du Langon réalisée en 2008 montre que ce type d'activité a pu exister dès l'époque gallo-romaine. Le développement relativement récent des analyses malacofauniques dans des contextes historiques le long du littoral atlantique français montre que dans certains villages localisés à la limite d'anciens marais, des niveaux composés quasi exclusivement d'huîtres sont localisés en dehors de zones d'habitat. La composition de ces dépôts contraste nettement avec les rejets alimentaires des populations côtières locales. En effet, pendant l'Antiquité et le Moyen Âge, ces populations exploitaient aussi des patelles 


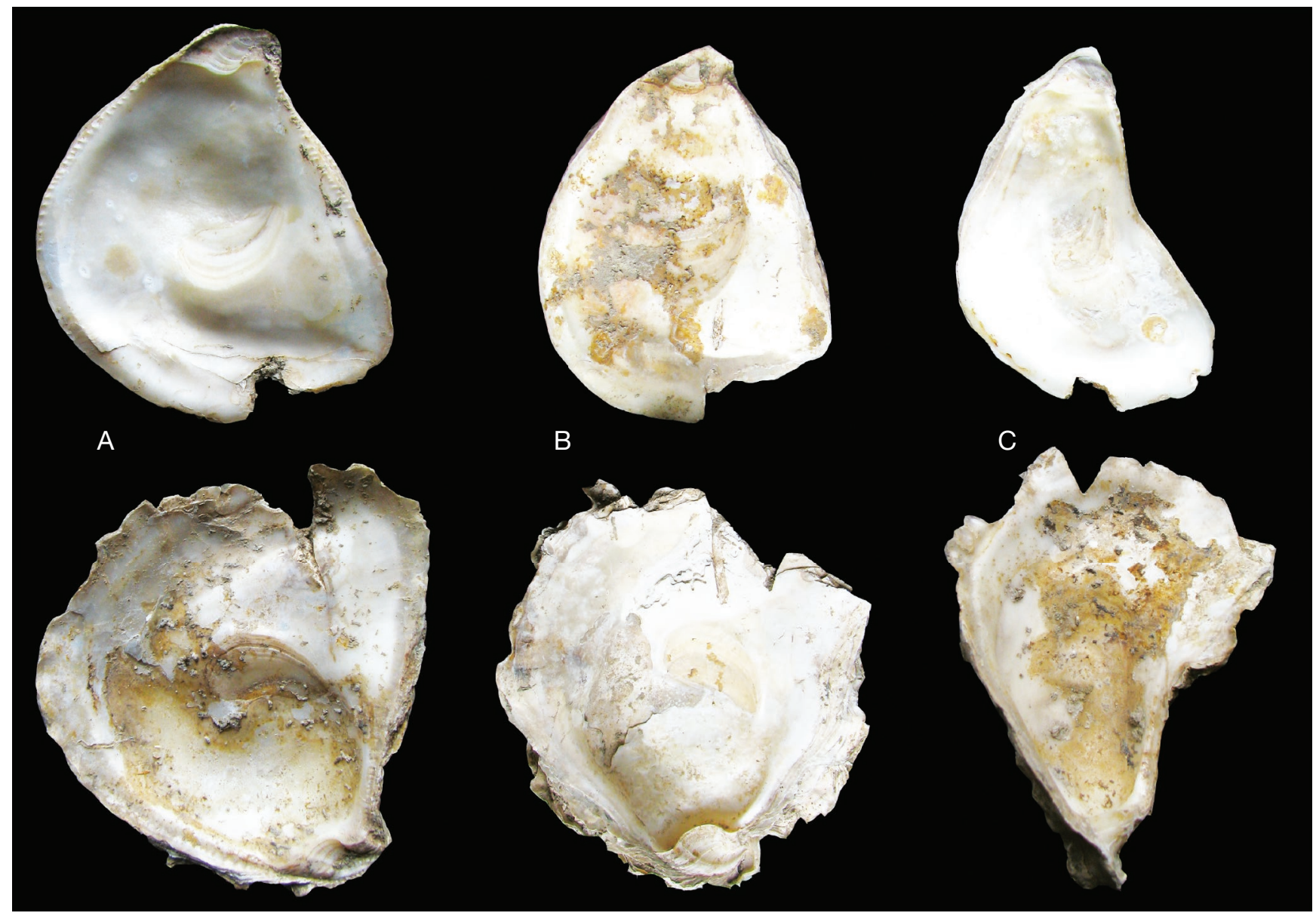

FIG. 11. - Stigmates d'ouverture sur la face interne d'huîtres trouvées jointives: A, L=92 mm; B, L=120 mm; C, L=107 mm (C. Dupont, CNRS).

Patella sp., des bigorneaux Littorina littorea, des pétoncles Chlamys varia, des coques Cerastoderma edule (Linnaeus, 1758), des monodontes Phorcus lineatus (da Costa, 1778), des moules Mytilus edulis, des scrobiculaires Scrobicularia plana (da Costa, 1778) et des palourdes Ruditapes decussatus (Linnaeus, 1758)(Dupont pers. comm.).

Il serait intéressant de savoir si ces sites à exploitation quasi exclusive d'huîtres sont contemporains. Sur les sites archéologiques éloignés de la mer, les dépôts médiévaux semblent montrer de plus faibles quantités de ressources marines que ceux attribués à l'époque gallo-romaine. Bien que cette différence puisse en partie s'expliquer par des biais taphonomiques, des fouilles moins systématiques de rejets alimentaires dans les contextes médiévaux, ou des contextes de dépôts différents, d'autres hypothèses peuvent être proposées, comme celle d'un décoquillage massif des huîtres, plus visible au Moyen Âge. À quoi cette exploitation massive d'huîtres peut-elle correspondre? Reflète-t-elle une demande croissante des populations urbaines vis-à-vis des ressources marines? L'extraction de la chair de la coquille limite le poids du produit transporté. De nouveaux procédés de conservation, comme l'utilisation du sel, sont-ils des initiateurs de cette nouvelle activité? L'évolution des goûts a-t-elle pu participer à ce développement? Les sources historiques manquent à ce sujet.
Les investigations actuelles ne nous ont pas permis de suivre l'itinéraire de la chair des huîtres de leurs lieux de production vers les places de consommation, du fait de la méconnaissance des jalons économiques et politiques locaux, et de la disparition des contenants de bois ou de céramique. Comme pour l'étude du sel, nous sommes ici au début d'une réflexion qui prendra appui sur les sources historiques. Souhaitons à ce site un classement au titre des monuments historiques, pour lui éviter des fouilles préventives.

La question évoquée des filières de production et d'achalandage, le rôle des ordres religieux et comtal, celui des négociants, marchands et marins qui concouraient à fournir les marchés du pays, reste, pour l'essentiel, à étudier.

\section{Remerciements}

Ceux-ci s'adressent principalement à Didier le Gouestre, chef du laboratoire d'archéologie du Service Régional d'Archéologie des Pays de la Loire, qui a accueilli C. Dupont au sein de ses locaux lors de l'analyse malacofaunique, ainsi qu'à Y. Gruet, qui nous a apporté une aide considérable dans les apports bibliographiques et relu ce manuscrit. Nous tenons aussi à remercier C. Lefèvre, M. Sternberg et les relecteurs anonymes pour leurs remarques et corrections. 


\section{RÉFÉRENCES}

BAUDOIN M. 1912. — La chaussée et la butte d'huîtres de Beauvoirsur-Mer (Vendée). Bulletins et Mémoires de la Société d'Anthropologie de Paris, VIe s. 3 (3-4): 222-242.

BAUdoIn M. 1916a. — Démonstration d'un Monument cultuel, du type des Tertres animaux en forme de Serpent et d'origine Nordique, aux Buttes coquillières des Chauds, commune de Saint-Michel-enL'Herm (Vendée) [actions humaines exécutées sur huitres entières]. Bulletin de la Société Préhistorique Française 13 (7): 369-384.

Baudoin M. 1916b. - Démonstration d'un Monument cultuel, du type des Tertres animaux en forme de Serpent et d'origine Nordique, aux Buttes coquillières des Chauds, commune de Saint-Michel-en-L'Herm (Vendée) [actions humaines exécutées sur huîtres entières] (suite). Bulletin de la Société Préhistorique Française 13 (8): 503-516.

BAUdoin M. 1916c. - Démonstration d'un Monument cultuel, du type des Tertres animaux en forme de Serpent et d'origine Nordique, aux Buttes coquillières des Chauds, commune de Saint-Michel-en-L'Herm (Vendée) [actions humaines exécutées sur huîtres entières] (suite). Bulletin de la Société Préhistorique Française 13 (9): 562-576.

Begouen C. 1937. - À propos des huîtres de Saint-Michel-enl'Herm. Revue du Bas-Poitou 50 (1): 85-91.

BERNARD E. 1994. - Gisements salicoles du Sud-Vendée. Rapport de prospection thématique $n^{\circ}$ 94-080. DRAC des Pays de la Loire, Nantes, $45 \mathrm{p}$.

CavoleaU J.-A. 1844. - Statistique ou description générales du département de la Vendée. Robuchon, Fontenay-le-Comte, 944 p.

ClerC L. 1828. - Manuel de l'amateur d'huîtres, ou l'art de les pêcher. L'Éditeur, Paris, 79 p.

DuCOS T. 1853. - Le Règlement sur la police de la pêche maritime côtière dans le 4 e arrondissement maritime. Imprimerie impériale, Paris, $95 \mathrm{p}$.

Duhamel DU Monceau H. 1769. — Traité Général des Pesches et Histoire des Poissons ou des animaux qui vivent dans l'eau. Section II.

DupONT C. 2006. - La malacofaune de sites mésolithiques et néolithiques de la façade atlantique de la France: Contribution à l'économie et à l'identité culturelle des groupes concernés. British Archaeological Reports, International Series 1571. Archeopress, Oxford, $439 \mathrm{p}$.

Dupont C. \& Bougeant P. 2008. - Des coquillages soumis à une source de chaleur aux huîtres trouvées valves jointes (la rue de l'industrie, Langon, Vendée) in Hervé M.-L. (éd.), Le Langon (Vendée): Rue de l'Industrie. Rapport final d'opération, 97-121.

FERDIÈrE A. 1988. - Les campagnes en Gaule romaine. Tome2: Les techniques et les productions rurales en Gaule (52 av JC-484 ap JC). Errance, Paris, $284 \mathrm{p}$.

GAMBIER J. \& RobuCHON J. 1936. — Encore les Buttes Huîtrières. Revue du Bas-Poitou 4: 283-294.

GEORGE J.-P. \& NÉDÉLEC C. 1991. — Dictionnaire des engins de pêche. Ifremer; Éditions Ouest-France, Rennes, 278 p.

GRELON M. 1978. - Saintonge, pays des huîtres vertes. Rupella, la Rochelle, $364 \mathrm{p}$.

Gruet Y. \& Prigent D. 1986. — Les buttes de St-Michel-enl'Herm (Vendée) : caractère de la population d'huîtres (Ostrea edulis, Linné) et sa faune associée. Haliotis 15: 3-6.

Guillet J. \& Guillet R. 2008. - L'ostréiculture en Bretagne de 1850 à nos jours. Coop Breizh, Spézet, $321 \mathrm{p}$.

Gutiérrez-Zugasti I., Andersen S. H., Araujo A. C., Dupont C., Milner N. \& Monge-SoAres A. M. 2011. — Shell midden research in Atlantic Europe: state of art, research problems and perspectives for the future. Quaternary International 239: 70-85.
Hughen K. A., Baillie M. G. L., Bard E., Beck W. J., Bertrand C., Blackwell P. G., Buck C. E., Burr G., Cutler K. B., Damon P. E., Fairbanks R. G., Friedrich M., Guilderson T. P., Kromer B., McCormac G., Manning S., Bronk Ramsey C., Reimer P. J., Reimer R. W., Remmele S., SOUthon J. R., Stuiver M., Talamo S., Taylor F. W., Van der Plicht J. \& WeYHENMEYER C. E. 2004. - Marine04 marine radiocarbon age calibration, 0-26 Cal Kyr BP. Radiocarbon 46 (3): 1059-1086.

Lacroix L. 1942. - La Baye de Bretagne. Histoire de la Baie de Bourgneuf et de son littoral. S. Pacteau, Luçon, $356 \mathrm{p}$.

Le Masson du ParC F. 1730. - Procès verbaux d'inspections des pêches sur les côtes de France. Archives de la Marine.

LeVAsseur O. 2005. - Brève histoire de la consommation des produits de la mer (XVIe - XIXe siècles), in XVIIème congrès de l'Association internationale des sociologues de langue française, $C R$ 17 Sociologie et anthropologie de l'alimentation, Tours, 5-9 juillet 2004., 13 p.

LEVESQUE J. 1982. - Granville et la Pêche au temps des Terre-Neuvas. Editions Charles Corlet, Condé-sur-Noireau, 143 p.

LISTER M. 1873. - Voyage de Lister à Paris en MDCXCVIII. Société des Bibliophiles, Paris, 143 p.

Marchand G., Dupont C., Oberlin C. \& Delque-Kolic E. 2009. — Entre «effet réservoir» et «effet de plateau » : la difficile datation du Mésolithique de Bretagne, in Crombé P., VAN Strydonck M., Sergant J., Bats M. \& Boudin M. (éds), Proceedings of the international congress "Chronology and Evolution in the Mesolithic of NW Europe", Brussels, May 30-June 12007. Cambridge Scholar Publishing, Cambridge: 307-335.

Mourain dE SourdeVAL C. 1864. - Lancien port de Beauvoir-surMer. Annuaire de la Société d'Émulation de la Vendée 10: 231-239.

RIVIÈRE A.-A. 1834. - Notice sur les terrains d'atterrissements et en particulier sur les Buttes de Saint-Michel-en-l'Herm. Dictionnaire pittoresque d'Histoire Naturelle 8: 1-44.

Rousseau J. 1963. - Beauvoir-Sur-Mer. Chroniques Paroissiales Nouvelle Série 13: 1-469.

Rouzeau N., Bernard E., Pascal J. \& Gruet Y. 2002. — Sauneries et briquetages. Essai sur la productivité des établissements salicoles gaulois du Centre-Ouest atlantique d'après l'étude du gisement de Nalliers (Vendée), in WeLLER O. (éd.), Internationale Archäeologie, ASTK 5. Table ronde du Comité des Salines de France, Paris, mai 1998. Verlag Marie Leidorf GmbH, Rahden: 99-124.

SORET L. 1985. - À Beauvoir-Sur-Mer, deux énigmes: la chaussée huîtrière et la cuve cimentée. Annuaire de la Société d'Émulation de la Vendée 132e année: 13-33.

SORET L. 1994. - Beauvoir-Sur-Mer, sa contrée et la baie de Bourgneuf à travers les âges. OFFSETS édition, La Mothe Achard, $544 \mathrm{p}$.

Stuiver M. \& Reimer P. J. 1993. - Extended 14C data base and revised CALIB 3.0 14C age calibration program. Radiocarbon 35: 215-230.

Ters M. \& Viaud J.-M. 1983. - Notice de la carte géologique de Challans. BRGM, Paris, $62 \mathrm{p}$.

VERGER F. 1959. - Les buttes coquillières de Saint-Michel-enl'Herm. Norois 21: 35-45.

VERGER F. 1968. - Marais et Wadden du littoral français: étude de Géomorphologie. Biscaye Frères, Bordeaux, 541 p.

VERGER F. 2005. - Marais et estuaires du littoral français. Belin, Paris, 335 p.

Villalobos C. \& MÉnanteau L. 2006. — Paléoenvironnements et techniques de production du sel marin (par ignition ou insolation) durant l'Antiquité: le cas des baies de Bourgneuf (France) et de Cadix (Espagne), in Hocquet J.-C. \& Sarrazin J. L. (éds), Le Sel de la Baie. Presses Universitaires de Rennes: 87-101. 